DOI: $10.30520 /$ tjsosci. 988564

\title{
FINANCIAL VIABILITY OF CRYPTOCURRENCIES IN PAKISTAN
}

\author{
Zahra Ahmed Sheikh ${ }^{1}$
}

\begin{abstract}
The globally acknowledged accelerating crypto hype has put a lot of work on researchers and analysts. This new marvel of digital currencies requires a lot of attention, for it to become conventional worldwide. Virtual coins or cryptographic forms of money utilize encryption framework, so called the Block Chain technology, that manage the formation and supply of coins and exchanges must be recognized from a financial examination point of view. Subsequently, it is essential to inspect which social, money related \& macroeconomic components decide its cost with a specific end goal to know the degree and outcomes of the economy. This paper aims to study different internal and external factors that affect cryptocurrencies' prices. A sample of four digital coins with largest market capitalization has been selected. Daily price data from the years 2015 to 2020 of Bitcoin with other altcoins such as Ethereum, Ripple and Litecoin has been taken. Internal factors consist of demand and supply variables and also the attractiveness associated with its increasing hype. Other factors include KSE-100 Index (Karachi Stock Exchange), USD-PKR (Dollar to Pakistani Rupee) exchange rate and oil prices from PSO (Pakistan State Oil). ARDL analysis has been done to study the effect of these factors on the prices of crypto coins. Our analysis shows that circulating supply has a significant effect on Ethereum and Ripple prices in the long run. Attractiveness has been significant on the prices of Ethereum only.
\end{abstract}

\section{Keywords}

Cryptocurrency, Bitcoin, Altcoins, Attractiveness, Demand, Supply, Macroeconomic and financial developments, Price.

\footnotetext{
${ }^{1}$ Visiting Lecturer, Bahria University Islamabad Campus, Pakistan. Email:rafiquezahra2001 @ gmail.com. Orcid ID 0000-0001-8494-3097
} 


\section{INTRODUCTION}

\subsection{Crypto Ecosystem}

In 2008 Satoshi Nakamoto, an unknown person/group floated the idea of an entirely new entity, digital currencies (Nakamoto, 2009). Cryptocurrencies are considered as those universally accepted marvels whose prime aim was to facilitate electronic payments without the involvement of a trusted third party (Abeer ElBahrawya, 2017). Cryptocurrencies exchanges don't rely on central banks for their payment execution but they are based on decentralized computer system, the block chain innovations to endorse exchanges and advance cash supply (Yermack, 2015). The extraordinary advancement of monetary standards has stirred the intrigued of governmental institutions which are stressed about financing criminal action and their effect on the stability of national financial frameworks. That is why part of scholarly inquire about has been conducted on cryptocurrencies to explore if they may be cash or theoretical resource (Baek \& Elbeck, 2015).

Cryptocurrencies are computerized tokens that can be exchanged online, using cryptographic hashing and advanced marks to check exchanges and maintain a strategic distance from two fold spending of the same type of coin. This idea gave the world of an insight into what other forms of medium of exchange can operate without the intervention of a regulatory body, thus bringing the transaction cost to its lowest. Initially only the Bitcoin was in circulation but different types of digital currencies are present on different coin exchanges by now. These digital securities have become part of our investment world, the same way as the stocks and bonds are. The need of the day is to better understand this new phenomenon to fully utilize its usage. This study will focus at the various factors that affect the prices of these digital currencies. This will help to identify the components that can help in stabilizing the extreme volatility associated with them. This volatility acts as a barrier to make cryptocurrencies globally conventional (Pavel Ciaian, 2016). This paper will be helpful in understanding the financial viability of cryptocurrencies in Pakistan.

\subsection{Characteristics of Cryptocurrency}

\subsubsection{Decentralized no central authority}

In conventional fiat monetary form, central bodies and banks control the budgetary framework. But with cryptocurrencies these exchanges can be prepared and approved by a conveyed and open organize that is claimed by no one (Baur \& Dimplf, 2021). Cryptocurrencies are based on distributed network technology. These distributed network computers exist all around the world and are known as nodes. Blockchain, also called the public distributed ledger records the transactions verified by these nodes through cryptography. Every node in the network has the same shared copy of these recorded transactions so there is no need to have a central authority that approves their legality. Aside from the other major benefits of cryptocurrencies and blockchain innovation, understanding the centralized believe issue alone could be an enormous sufficient advancement to grant cryptocurrencies their remaining control. 


\subsubsection{Irreversible and immutable}

The irreversible and unchanging highlights of cryptocurrencies suggest that it is outlandish for anyone but the proprietor of the particular private key to move their transactional data off the blockchain recorded data base. Since this record is made public and with the help of cryptographic security, it is extremely difficult to corrupt the entire network.

\subsubsection{Anonymous}

Since there is no central body governing the system, the users don't need to verify their identities. They can securely execute their transactions using the open source peer to peer network. This system employs a private key and public key system to authenticate, verify and securely record these transactions on the network. This implies that any user can make unidentified accounts and computerized wallets to safely execute their transactions on decentralized framework.

\subsubsection{Limited supply and scarcity}

Cryptocurrencies have a restricted and predetermined supply that has been written in its algorithm code when they are created. With cryptocurrency, in any case no person or consortium is able to influence the supply of the money or apply noteworthy impact over it without the endorsement of the lions share. Numerous top cryptocurrencies have a fixed and limited supply thus making them deflationary in nature. Any increment within the request or selection of it will cause a comparing increment in its price.

\subsubsection{Cryptography}

Cryptography advanced out from the demand of secure communication during World war II. Easily readable data was encrypted into scrambled code. Since then cryptography has been in use in different forms. In todays computerized world cryptography is based fundamentally on computer science and scientific hypothesis. Two fundamental components of cryptography apply to cryptocurrencies, Hashing and Computerized signatures. Hashing confirms information, keeps up the structure of the blockchain and encodes peoples account addresses and proceedings. It also creates cryptographic problems for block mining process. Computerized signatures permit a person to demonstrate that they claim a bit of scrambled data without uncovering it. With cryptocurrencies this innovation is utilized to sign financial contracts. It affirms that an account proprietor has concurred to the exchange.

\subsubsection{Blockchain technology}

Blockchain is the decentralized, open and immutable transactional record of cryptocurrencies. Completed and verified transactions are stored in blocks and added to the previous set of mined blocks in chronological order. This data base of mined blocks is visible to all the nodes in system, which permits them to track exchanges without the need of verification from a central authority. This innovation makes a record that can't be changed without the assentation of the rest of the nodes. The blockchain concept is credited to bitcoins originator, Satoshi Nakamoto. This concept has been the motivation for other applications as well. 


\subsubsection{Block Mining}

Block mining is the process of adding new blocks to the blockchain network. Mining requires very high processor computers to solve mathematical problems thus validating the transactions and making chains of blocks with immutable data. The speedier a miner's equipment can handle the scientific puzzle, the more likely it is to approve a transaction and gain crypto compensate.

A cryptographic money is a digital or virtual cash that utilizations cryptography for security. A cryptographic money is really hard to fake due to its security highlight. Rather than depending on customary money related organizations that confirm and assurance our exchanges, cryptographic money exchanges are checked by the user's personal computers signed into the cash's system. Since the cash is secured and encoded, it ends up really hard to extend the money supply over a predefined algorithmic rate.

\subsection{Crypto Currencies Industry Sectors}

There are four sectors in which virtual currencies operate (Rauchs, 2017).

\subsubsection{Crypto Exchanges}

We define digital currency exchange or coin exchange as any medium that enables clients to purchase and offer digital currencies for different types of cash or resources (Rauchs, 2017). Crypto Exchanges give administrations to purchase and offer cryptographic and digital forms of money. Coin exchanges assume a fundamental part in the digital money economy by offering a commercial center for exchanging, liquidity, and value revelation. The first use case of cryptocurrency were exchanges and the highest number of employees work in this sector of the crypto industry (Morisse, 2015).

\subsubsection{Wallets}

Cryptographic money wallet is a protected computerized wallet used to store, send, and get advanced cash like Bitcoin and other Altcoins. Most coins have an official wallet or a couple of authoritatively prescribed outsider wallets. Keeping in mind the end goal to utilize any digital currency, we should utilize a cryptographic money wallet. Cryptographic money itself isn't really kept in it. Rather, a private key which is a secure computerized code known just to you and your wallet, is put away that shows responsibility for open key, an open advanced code associated with a specific measure of money. So your wallet that stores your private and open keys actually enables you to send and get coins.

\subsubsection{Transactions in crypto market}

All digital currency frameworks have a coordinated payment system to process exchanges designated in the local token. While the guarantee of these frameworks is that clients can autonomously execute on these systems (Rauchs, 2017). Payment organizations for the most part go about as passages between clients of blockchain exchange framework. To summarize, this sector includes all transactions being done in crypto spot and derivatives exchanges. 


\subsubsection{Cryptocurrency Mining and the Block Chain}

Block Chain technology is the foundation of almost all cryptocurrencies. It is a technical process which involves finding the right codes to locate valid transactions of cryptocurrencies and adding them as a block to the previously existing blocks, hence forming a chain of all proceedings. This aids to maintain the record of all the transactions done in cryptocurrencies without the presence of any regulating body. Anybody with access to the web and appropriate equipment can take an interest in mining. The miners are then rewarded for their contribution. The mining part has created in a short time allotment from a side intrigue development performed on personal computer into a specialist and capital-genuine industry with its own specific regard chain (Rauchs, 2017).

The record is freely accessible for everyone to examine the whole blockchain network. The client's anonymity remains in place and they are recognized just by utilizing their open key as an address. The transaction data is encoded as well. Invalid exchanges are dismissed and are excluded from the previously authenticated mined blocks. Endeavors for vindictive changes in the exchanges will require rehashed computation of the confirmation of work for the mined blocks (Karafiloski, 2017).

\subsection{Crypto Currency Versus Standard Currency}

The three main functions of the money are medium of exchange, unit of account and store of value. In order to investigate whether CC can replicate standard currency we need to look at its characteristics. According to Folkinshteyn (2015), Bitcoin, the most popular kind of CC can act as a perfect medium of exchange owing to its low transaction cost and rapid transaction time. Since it is virtual with no physical existence, it does not require production cost like the standard fiat currency. The other plus points in favor of it are the privacy and anonymity, infinite divisibility and no pressure of inflation.

There are also some characteristics that go against its usage as a medium of exchange which are summarized here. Rainer Böhme (2015) believed that security is a main threat to use CC as medium of exchange because there is no oversight institution in control. Also cyber-attacks cannot be ignored because of the anonymity factor. They need proper expertise and technical equipment to be adopted hence adding the cost. Whereas, the standard currency can operate without such an adoption cost. Finally, Yermack (2015) negates this idea of virtual currency system by adding that this virtual system is not easily understood and globally accepted. The most important of all is its extreme price volatility which obstructs it being used as a conventional currency. Each time you swipe your credit or platinum card, your own data is appended, and organizations, banks and governments can utilize this information to track your exercises. Cryptographic money exchanges convey no close to home data, except if you include it yourself. This security likewise drastically diminishes the odds of fraud. Consistent access to your records. Customary records can be embellished or solidified, however since advanced cash exists outside the directions and laws that enable this to occur, it's extremely uncommon to be not able to access your coins. Singular cryptographic forms of money are advanced and can't be falsified or turned around subjectively by the sender. Conventional banks charge expenses to process exchanges. With digital money being traded over the web, there are practically no exchange expenses. There are around 2.2 billion individuals with access to the Internet or cell phones who do 
not approach a customary trade. For these individuals, the digital money is great. Bitcoin, Ethereum, and Blockchain have moved towards becoming piece of the innovation publicity cycle in 2017. Bitcoin keeps on setting untouched highs for quite a while, while Ethereum guarantees to utilize Blockchain to interrupt every monetary instrument and business forms. Baur \& Dimplf (2021) investigated whether Bitcoin can replicate standard money forms. They suggested that since Bitcoin is not backed by Gold or any regulatory authority it cannot act as a medium of exchange and has a restricted utilize as a risk diversifier. Rather, keeping its deflationary aspect in mind it does exhibit the store of value element.

\subsection{Problem statement}

The main problem associated with the digital currencies is their immense volatility. The elements that determine their prices are still ambiguous. Although this problem is not only associated with crypto prices in US Dollars, Yen and British Pounds but also with other currencies. This study will examine the effect of relevant factors on crypto prices in Pakistan. The latest problem that has recently emerged is the restriction on crypto use by State Bank of Pakistan. Cryptocurrencies have been widely and excessively used by a lot of other developing and developed economies. As of late, significant saving money foundations and innovation organizations, for example, Intel, Barclays and Walmart have put their opportunity and cash into the guarantee of cryptographic forms of money like Bitcoin and Ethereum. This has prompted nations with debilitating monetary standards to embrace computerized money to replace customary notes that have devalued. A portion of these early adopter nations incorporate Brazil, Colombia, Turkey and Venezuela. Wealthy nations are likewise investigating receiving digital money as lawful delicate. As indicated by a report by Bloomberg, the National Banks of Japan, European Union and Holland are at present directing exploration ventures and trials on advanced monetary standards. The digital money unrest is likewise spreading to India, where head of state has lessened flow of money bills to guide the nation towards electronic installment endorsement. The Reserve Bank of India is currently investigating the most up to date wave without bounds digital money. Government of Pakistan ought to put an effort in bringing together all the resources to look for a set of regulations under which the crypto system can operate without the fear of its illegal usage.

This study intends to apprehend

- The immense volatility of the prices of different virtual currencies, and identification of the variables that affect their prices.

- Using digital currencies in place of standard currency or as another mode of payment.

- The analysis of the crypto ecosystems of Pakistan.

- The restrictions on the use of cryptocurrencies in Pakistan by State Bank has provoked to investigate whether crypto coin market has a significant impact on an economy and their exclusion from the current monetary system can be a big set back towards a country's growth. 


\subsection{Research objectives}

The key objectives of this paper is to:

- Examine the effect of crypto supply on crypto price.

- Examine the effect of crypto demand on its supply

- Explore the efficacy of attractiveness on virtual currency prices in Pakistan.

- Investigate the effect of prices of KSE-100 Index on the prices of cryptocurrencies.

- Investigate the effect of exchange rate (USD-PKR) on digital currency prices.

- Investigate the effect of oil prices in Pakistan on crypto prices.

\subsection{Research questions}

This study aims to examine:

- What is the effect of demand and supply on the prices of cryptocurrencies?

- What is the impact of attractiveness on crypto coins' value?

- What is the role of macroeconomic developments on crypto currencies prices?

- Why cryptocurrencies should be part of the monetary system?

\subsection{Significance of the study}

Financial crisis of 2008, political manipulation and continuous devaluation of standard money prompted nations to switch to a digital financial system which can operate without the involvement of a regulating body. This study will smooth the way for law makers to realize the importance of the globally emerging phenomenon of cryptocurrencies. This will help the policy makers to conduct laws of trade accordingly. The prosperity of a country is the ultimate goal of its state officials and it lies in the financial growth of its economy. Analysis of the causal effect between the macro financial developments of a country and virtual currencies will determine the significance of this new digital form of money on a country's economic growth. Because of the immense price flicks cryptocurrencies act like a speculative investment. Investors can benefit from this research in deciding which crypto coin is the best for them to plough money into. There is not much research done in Pakistan on digital currencies so this study will act as a mile stone for future researches. 


\subsection{Research gap}

The effects of demand supply equilibrium, attractiveness and global macro indicators on the dollar price of Bitcoin have already been studied by Pavel Ciaian (2016). This study will investigate whether the same relation holds for other crypto coins which are Etherium, Litecoin and Ripple. The denomination used in the analysis is PKR i.e. Pakistani Rupee. Previous studies like Selmi (2015) used foreign exchange and inflation as macro financial indicators effecting Bitcoin price. Second contribution of this paper will be the usage of Pakistan state oil prices, Karachi stock exchange (KSE100) Index and Exchange Rate between US dollar and Pakistani rupee (PKR) as proxies to macroeconomic and financial developments, to study their effects on crypto currencies prices.

\section{LITERATURE REVIEW}

\subsection{Previous Studies}

Since its development in 2009, a considerable measure of investigation on this marvel has been seen up till now. The cryptographic money showcase has seen an unmatched level of enthusiasm from financial specialists in 2016. Bitcoin, otherwise called the dad of every single computerized cash, has risen in excess of 1,500 percent since the beginning of 2017. There have been a lot of concentrates, for example Selmi (2015) and Katsiampa (2017), breaking down the eventual fate of Bitcoin and its instability. Bitcoin is as of now exchanging at $\$ 16,000$. Bitcoin cost was at $\$ 1,000$ toward the start of the year. Katsiampa (2017) checks the unpredictability of Bitcoin through an examination of GARCH models and finds that the AR-CGARCH display gives the best fit. He complements that the market is greatly unusual. Selmi (2015) contemplate day by day Bitcoin costs sending an ideal GARCH model and demonstrate that the instability is dropping contrasting pre-and post-2015 information. They identified critical skewness in the Bitcoin advertise where the costs are controlled more by negative than positive stuns. In like manner, Dyhrberg (2015) utilizes the deviated GARCH philosophy and infers that Bitcoin can be utilized as a supporting instrument against stocks in the Financial Times Stock Exchange Index and against the American dollar for the time being.

Then again, Abeer ElBahrawya (2017) analyzed working of the entire market of around two thousand digital forms of money between April 2013 and May 2017. They reasoned that cryptographic forms of money travel every way consistently and their market capitalization is always mounting while other scientific properties of the market have been comparable for quite a long time. uniquely, piece of the pie dispersion and the turnover of cryptographic forms of money continue as before. Sovbetov (2018) uses ARDL approach to document the determinants of five different crypto prices. Market factors such as beta, trading volume \& volatility seem to be vital determinant both in the long and short run. On the other hand, attractiveness conjointly matters in terms of their value determination, however solely in long run. Moreover, SP500 appears to possess weak positive long term effect on crypto prices and completely insignificant in short term analysis.

There are two types of factors that had been studied throughout the literature which effect the pricing of Bitcoin. First is the attractiveness and the other one macroeconomic indicators. The first type has been suggested by Kaminski (2014). He studied the effects of twitter as a gauge to determine Bit price. Another one in the league is the study by Wilson (2015) who scrutinized trend data of google 
to measure interest in bitcoin, which subsequently effect its price. The second type of factor analyzed is macroeconomic indicators. These internal and external drivers have been studied by Kristoufek (2013).

Wijk (2013) investigates the factors that determine the value of fiat money and hence derived a model to find out the value of Bitcoin. Up till 2013 not much research was done in this regard. The eight variables used in this analysis include price of Bitcoin in US dollar as stated by Mt Gox, which is one of most leading trader of Bitcoin. Other variables are Dow Jones index, FTSE 100 index, Nikkei 225 index, Brent oil price, WTI oil price, CMCI oil and the two exchange rates US/EUR and US/YEN. Time frame taken was from $19^{\text {th }}$ July 2010 to $13^{\text {th }}$ June 2013. This includes 759 observations. Data has been modified to be used for analysis. ADF test done to check for stationarity. The first difference has been taken of all the variables that are non-stationary. Analysis has been done through OLS using E-Views 7. F-Test has also been done to check the model. Godfrey LM test has been done to check for any chances of serial correlation. ECM technique which is called error correction model has been used to study both the long run and short run effects. ARDL model derived from it and OLS used to estimate the model. After the final perusal it is concluded that Dow Jones Index, US-EUR exchange rate and WTI oil price significantly affect the Bit price in the long run. Daily analysis has been done on the time series of volume and velocity of bitcoin trade, volume is to velocity ratio, number of transactions, hash rate, google trends and gold price. Kristoufek (2013) recorded Bitcoin price from four main exchanges of that time. These exchanges are Bitfinex, Bitstamp, BTC-e and Mt Gox. Data time frame is 2011 to 2014. Wavelet methodology has been deployed. The analysis of the impact of Chinese market on dollar market has also been recorded. The results show that there is no proof of impact of Chinese market on dollar market. Money supply, use in trade and price level have an impact on Bitcoin price. Attractiveness leads to increased demand of it.

Selmi (2015) in November deployed ARDL Bound testing method and VEC Granger causality tests to determine the factors that affect the price of Bitcoin both in the long and short run. The variables studied here include investors attractiveness, economic, macroeconomic and financial indicators and technical drivers. Economic indicators used here are exchange trade ratio, monetary velocity and output volume. Gold price and Shanghai stock market index are used as proxies for macroeconomic and financial indicators. Technical drivers are gauged through Hash rate. Time frame of the data used is 2010 to 2014. Dummy variable induced here that indicates bankruptcy of Chinese trading company which is 1 for 2013 and 0 otherwise. The results of ARDL show that attractiveness is a significant driver of the bitcoin price valuation. 10 percent increase in exchange trade ratio brings about 0.32 percent of bitcoin price. The technical driver, hash rate does effect the price of Bitcoin but with a small magnitude. The rest of the drivers have no significant effect on Bitprice.

Pavel Ciaian (2016) compared characteristics of Bitcoin with those of standard currencies. They inferred that price volatility is the only feature that stands out. Because of its extreme volatility Bitcoin cannot replace the conventional modes of payment. Hence the drivers of its price movements had to be analyzed. Daily data from 2009 to 2014 time period has been taken. The explanatory variables of the model include exchange rate US/EUR, size of bitcoin economy, velocity of its circulation, total stock of bitcoins, attractiveness and macroeconomic and financial indicators. Macroeconomic indicators include oil prices and Dow Jones index. A multivariate Vector Auto Regressive model has 
been specified. Stationarity of the time series has been checked through the Augmented Dickey-Fuller test and Phillips Perron test. Akaiki Information Criterion (AIC) decides the lags used for each variable. Johansen's cointegration method has been deployed to study the long term model. Maximum eigenvalue test and trace test decide the number of cointegrated vectors. Vector error correction method has been used to correct the cointegration. Langrange-Multiplier (LM) test for autocorrelation of the residuals, Jarque-Bera test and a test of stability of model have also been conducted. Estimation results conclude that Bitcoin attractiveness has a significant effect on Bitprice. Also the market forces of demand and supply influence the price of Bitcoin quite significantly. Whereas, the macro-financial indicators do not have a significant effect on bitprice in long run.

Poyser (2017) uses Bayesian structural time series analysis to identify the linkage between price of bitcoin and its determinants. This paper categorizes bitcoin price determinants into two groups which are internal factors and external factors. Internal factors include market forces of demand and supply whereas external factors consist of attractiveness and macro-financial indicators. The contribution of this paper which makes it stand out from the previous studies, is that it measures attractiveness across different countries. Exchange rate US/YUAN has been taken because most of the bitcoin transactions have been recorded in United States and China. Gold price has been taken as a financial indicator. Data time frame is 2013 to 2017. The estimation results show that gold price, exchange rate US/YUAN and investors attitude are inversely proportional to bitcoin price. Whereas positively related to sock index. Sovbetov (2018) studied price factors of five cryptocurrencies Bitcoin, Etherium, Dash, and Monero. 2010 to 2018 weekly data has been analyzed using ARDL technique. $\mathrm{He}$ also categorizes price factors into two subheadings, internal and external factors. Market forces of demand and supply have been taken as a main internal factor. External factors are attractiveness, legalization and macro-finance determinants (Interest rate, Stock market, gold prices). Short run and long run analysis has been done. ADF unit root test has been conducted to check for stationarity. First difference methodology has been adopted to rule out this problem. ARDL frame work which is also known as the Bound Testing Approach, has been deployed. Since the time series used contain I (0) and I (1) variables we can use this technique. In case there are I (2) variable this technique cannot be used. For hypothesis testing Wald analysis has been done. Breusch Godfrey LM test for serial correlation and Breusch-Pagan-Godfrey heteroscedasticity test has been applied. SIC value comes out to be minimum indicating that the model specified is very appropriate. Market return effects Bitcoin and Etherium significantly in the short run having multiplier value of 0.85 and 0.39 respectively. Volatility has significant effect on all currencies both in short run and long run. Trading volume significantly effects Bitcoin at $1 \%$ level of significance, and effects Etherium, Litecoin and Monero at $10 \%$ level of significance. Attractiveness effects all except Dash.

\subsection{Theoretical Framework}

Henry Thorton in 1802 surmised that more money supply in an economy leads to an increased inflation. This occurrence is known as the Quantity Theory of Money or QTM. It states that the total amount of money in circulation or the total money supply in an economy is directly proportional to the general price level of goods and services (Mankiw, 2006). When money supply increases the general price level also increases to compensate for a decrease in marginal value for money (Brue, 2001; Salvatore). 
There is a strong ongoing debate on whether cryptocurrencies can replicate standard currencies. Virtual monetary forms are proliferating across the globe. In a few sections of the world they are an indication of dissent against financial arrangement and expert. Somewhere else, they are a wellknown hypothesis resource. In some different spots, they are simply fascinating better approaches for payment, speculation and mix of genuine methods virtual life into real living (Sauer, 2016). All this happened in objection to financial crisis in 2008, fall back of the corrupt political systems and intervention of strict regulatory authorities into the money market. Cryptocurrency fulfills these needs by not driven by any standard third party laws and legislations and far away from the reach of monetary crisis. But the question arises as if virtual currencies perform the same functions as standard money does i.e. medium of exchange, unit of account and store of value. Pavel and Miroslav also suggested that the extreme price volatility of bitcoin acts as a hindrance to perform the above stated functions of money. These extreme variations in prices is due to the global hype created by investors and subsequently exaggerating the demand and supply function of it.

Cryptocurrencies can substitute standard forms of money if they follow their exact path and behave in the same fashion. For digital forms of money to replicate standard currency they should also follow the Quantity theory of money. This implies that increasing the circulating supply of crypto coins, causes a flick in its value. Other factors that affect the crypto prices are demand function, attractiveness and global macroeconomic and financial developments (Pavel Ciaian, 2016).

\subsection{Review of Variables}

\subsubsection{Demand and Supply variables}

Selmi (2015) Studied the effects of the market forces of demand and supply on the prices of Bitcoin. A year after Pavel Ciaian (2016) also studied the same relation. Both studies found the market forces of demand and supply to be significant.

\subsubsection{Attractiveness}

Attractiveness has been measured primarily by Kristoufek (2013) and then by Pavel Ciaian (2016). This variable has been the most significant of all, studied so far.

\subsubsection{Global macro and financial variables}

Wijk (2013) suggested the role of macro-economic and financial developments on the price of Bitcoin. Pavel Ciaian (2016) studied the combined effects of all the above mentioned variables on the price of Bitcoin. This study will examine the effects of all the above mentioned variables on the prices of four crypto coins that had been traded in Pakistan.

\subsection{Hypotheses of the study}

$\mathrm{H}_{1}$ : There is a relationship between crypto supply and the prices of digital currencies.

$\mathrm{H}_{2}$ : There is relationship between crypto demand and the prices of digital currencies. 
$\mathrm{H}_{3}$ : There is a relationship between attractiveness and crypto price.

$\mathrm{H}_{4}$ : There is a relationship between Stock prices and the value of cryptocurrencies.

$\mathrm{H}_{5}$ : There is a relationship between Exchange rate and cryptocurrencies prices.

$\mathrm{H}_{6}$ : There is a relationship between Oil prices and the value of crypto coins.

\section{RESEARCH METHODOLOGY}

This is a causal research in which we will be using secondary data for quantitative analysis. Daily crypto price data from the year 2015 to 2020 has been taken from Coingecko (2020), Coinmarket (2020) and Btcpk.net (2020). This study will be analyzed using ARDL method of estimation. There are over 2000 cryptocurrencies available in the market today. However, we are taking into account only four cryptocurrencies with the largest market cap which are Bitcoin, Etherium, Litecoin and Ripple. The model of this study has one dependent and three independent variables. Dependent variable is the price of four different cryptocurrencies denominated in Pakistani Rupee. Independent variables include crypto supply and demand, attractiveness and macroeconomic indicators. Supply and demand are gauged by market capitalization and trading volume respectively, Attractiveness is measured through percentage change in value respectively. Macro variables included in this study are Oil prices, Exchange rate and stock index of Pakistan. We may be able to conclude that Pakistan should also put its best effort to fully utilize this new phenomenon to accelerate its economic growth.

\subsection{Econometric Model}

On the basis of QTM and determinants of crypto price we make our model as follows

$$
\mathrm{p}_{\mathrm{t}}=\beta_{0}+\beta_{1} \mathrm{~s}_{\mathrm{t}}+\beta_{2} \mathrm{~d}_{\mathrm{t}}+\beta_{3} \mathrm{a}_{\mathrm{t}}+\beta_{4} \mathrm{k}_{\mathrm{t}}+\beta_{5} \mathrm{e}_{\mathrm{t}}+\beta_{6} \mathrm{O}_{\mathrm{t}}+\varepsilon_{\mathrm{t}}
$$

1. $\mathrm{t}=$ time subscript,

2. $\mathrm{p}_{\mathrm{t}}=$ Price of cryptocurrency

3. $\mathrm{s}_{\mathrm{t}}=$ Crypto supply

4. $\mathrm{d}_{\mathrm{t}}=$ Crypto demand

5. $a_{t}=$ Attractiveness

6. $\mathrm{k}_{\mathrm{t}}=$ Stock exchange price

7.e $e_{t}=$ Exchange rate price (USD/PKR)

8. $\mathrm{o}_{\mathrm{t}}=$ Oil price 
8. $\varepsilon_{\mathrm{t}}=$ error term

The prices of cryptocurrencies are denominated in Pakistani Rupee. we will be studying the effect of different economic factors on their prices in Pakistan's economy. Price data of four cryptocurrencies which are Bitcoin, Ethereum, Lite coin and Ripple, has been taken from Coingecko (2020). Demand for crypto coins will be gauged through volume data following Pavel Ciaian (2016). This data has also been taken from Coingecko (2020) and Coinmarket (2020). As demand has a positive relationship with price, the value of crypto coins should also increase with the increasing market cap of the relative coin market. We are using volume of the crypto economy as a measure to gauge the demand variable. Supply of crypto coins is measured by the market capitalization of crypto coins in circulation or being traded, following the quantity theory of money. Attractiveness is measured through the percentage change in crypto value. For Stock exchange variable we are taking KSE-100 Index which is Karachi Stock Exchange with 100 large market cap companies. Exchange rates of US Dollar and Pakistani Rupee has been used. Last variable used in our analysis is the oil price. PSO (Pakistan State Oil) prices have been utilized in this regard.

\subsection{Variable Description}

\subsubsection{Price of Cryptocurrencies}

This is the dependent variable in this study. According to QTM, increase in supply of the crypto coins, causes the price to rise and vice versa. Assuming that ' $\mathrm{V}$ ' and ' $\mathrm{T}$ ' remain constant throughout our analysis. The coefficients of attractiveness and macro indicators can have either positive or negative signs depending on the nature of positive or negative change in value of crypto price for attractiveness and the nature of the macro indicator used in our study. Study sample includes daily prices from the years 2015 to 2020 .

\subsubsection{Crypto Supply}

The first independent variable used in this study is crypto supply. Supply is a key monetary idea that portrays the aggregate sum of a particular decent or benefit that is accessible to purchasers. Supply can identify with the sum accessible at a particular cost or the sum accessible over a scope of costs whenever showed on a chart. This relates nearly to the interest for a decent or benefit at a particular value, all else being equivalent, the supply given by makers will rise if the value rises since all organizations hope to expand benefits. From supply we mean the circulating supply of the crypto coins. Pavel Ciaian (2016). Circulating supply is measured by the total size of the bitcoin economy i.e. their respective market cap. Market cap indicates the total number of crypto coins traded in the given time frame.

\subsubsection{Crypto Demand}

Demand in financial matters is characterized as purchasers' readiness and capacity to devour a given decent. The reverse connection among cost and amount requested of a decent is known as the law of demand and is regularly spoken to by a descending slanting line known as the demand curve. The law of supply and demand is a hypothesis that clarifies the communication between the supply of an asset 
and the interest for that asset. For the most part, low supply and high demand increment cost. Interestingly, the more prominent the supply and the lower the demand, the price tends to fall. The demand for crypto currencies has been gauged through the trading volume of the crypto coins. Volume of crypto coins indicates the total number of people engaged in its trade. We used it as a proxy to demand. The growing number of users indicate increasing demand for bitcoin and altcoins. This is also an independent variable of this study.

\subsubsection{Attractiveness}

Attraction is defined as an inclination towards something. Whatever we consider captivating, depends upon our discernment, the ability of our judgement and the available knowledge we have about it. From an investors point of view an investment is attractive if he has been receiving positive vibes about it. When everyone else in the market is weighing it up positively and favorably disposed towards it. Odean (2006) proposed that Investors decision is positively related to the expansion or reduction of rumination in the media. Lee (2014) found out that Bitcoin prices swelled because of the media hype and herding behavior. This infers that attentiveness may increase or decrease crypto prices based on the nature of either positive or negative inclination in news. For gauging the market attractiveness, it is important to look at the various factors affecting it. According to Peng (2009) one of the most important components influencing the market attractiveness is the growth rate. Růstu (2016) defines growth rate as a relative or percentage change in the value of something. This suggests that attractiveness of cryptocurrencies can be measured by the percentage change in its price.

\subsubsection{Global Macroeconomic and financial variables}

Wijk (2013) stresses the job of worldwide macroeconomic and budgetary improvement, caught by factors, for example, stock exchange lists, exchange trade rates, and oil costs measures in deciding Bitcoin cost. The effect of macroeconomic and budgetary pointers on Bitcoin cost may work through a few channels. For instance, stock trade files may reflect general macroeconomic furthermore, budgetary improvements of the worldwide economy. Great macroeconomic and budgetary improvements may invigorate the utilization of Bitcoin in exchange and trades and therefore fortify its interest, which may have positive effect on Bitcoin cost. Swelling and value lists are other sort of pointers catching imperative macroeconomic and monetary improvements. Oil cost is one of the primary wellsprings of request and cost weights, and it gives an early sign of expansion advancement. Additionally, the swapping scale may reflect swelling improvement and in this manner affect emphatically Bitcoin cost as showed previously. There could be likewise negative connection between a cash's cost and full scale money related markers. A decrease in the stock costs prompts remote speculators to offer the money related resources they hold. This prompts a deterioration of the basic cash, however may invigorate Bitcoin cost, if financial specialists substitute interest in stocks for interest in Bitcoin. For the most part, financial specialists' arrival on stock trade may catch opportunity expenses of putting resources into Bitcoin. Consequently, for this situation the stock trade lists are relied upon to be decidedly identified with Bitcoin cost. This study will examine the effects of stock exchange prices, exchange rate and oil prices on the value of not only Bitcoin but also on Litecoin, Ripple and Ethereum. 


\section{Stock Exchange}

A stock exchange, is where stock merchants and brokers can purchase and offer securities, for example, offers of stock and bonds and other budgetary instrument Karachi Stock Exchange 100 Index abbreviated as KSE-100 Index is a stock record going about as a benchmark to think about costs on the Pakistan Stock Exchange over a period. In deciding delegate organizations to process the list on, organizations with the most astounding business sector capitalization are chosen. This variable is a time series data set of the closing value of KSE-100 index.

\section{Exchange rate}

It is defined as conversion rate at which one currency will be traded for another. The conversion rate is a standout amongst the most vital determinants of a nation's relative level of financial wellbeing. Trade rates assume a crucial part in a nation's level of exchange, which is basic to practically every free market economy on the planet. There is a remarkable increase in GDP growth of a country with the price movements. If Exchange rate correlates with crypto prices, then this postulates that crypto prices act as a determinant of a country's economic prosperity. Exchange rate between US Dollar and PKR Pakistani rupee has been taken in this study.

\section{Oil price}

Inflation is a maintained increment in the general price level of goods and services in an economy over some specific time frame. It is a standout amongst the most critical issues in financial aspects. It impacts the loan fee on investment funds and the rate paid on home loans. Inflation likewise influences the level of pensions and benefits. High economic growth and low inflation go side by side. Oil prices detect early inflation in an economy they are taken as a measure of inflation. Daily prices of Pakistan State Oil have been taken for the time period of 2015 to 2020.

\section{ANALYSIS AND RESULTS}

Since we are dealing with time series data, stationarity of the variables has been checked through ADF test. First difference of the non-stationary variables has been taken to correct this problem. All models have been tested for serial correlation using LM test. According to Perasan table, the upper and lower bound values for explanatory variables are checked. If $\mathrm{F}$ statistic is less than the lower bound value, we conclude by saying that there is no long run association among the variables. On the contrary if the $\mathrm{F}$ statistic is greater than the upper bound value there exists a long run relationship among these variables and we estimate the long run model. Wald test suggests that there exists a long run association between variables in Ethereum and Ripple analysis only.

\subsection{Short run analysis}

\begin{tabular}{|l|l|l|l|l|}
\hline VARIABLES & Bitcoin & Ethereum & Ripple & Litecoin \\
\hline
\end{tabular}




\begin{tabular}{|l|l|l|l|l|}
\hline Supply & $-5.83 \mathrm{E}-07$ & $-4.36 \mathrm{E}-08$ & $-2.01 \mathrm{E}-10$ & $-1.17 \mathrm{E}-07$ \\
\hline Demand & $1.92 \mathrm{E}-08$ & $-1.17 \mathrm{E}-08^{* * *}$ & $6.22 \mathrm{E}-11 * * *$ & $-6.32 \mathrm{E}-09$ \\
\hline Attractiveness & -549.5794 & -11.07634 & -0.021265 & 2.067109 \\
\hline Stock Prices & -0.610845 & 0.595244 & -0.001575 & 0.104919 \\
\hline Exchange Rate & 13028.75 & -250.9274 & -0.838021 & 86.41514 \\
\hline Oil prices & -276.3957 & -4.606601 & $0.188296 * * *$ & -14.84495 \\
\hline Constant & 1757.680 & $-3.232487 * * *$ & -1.447546 & -31.72567 \\
\hline F-Statistic & $55.47676 * * *$ & $28.03311 * * *$ & $27.80098 * * *$ & $89.63480 * * *$ \\
\hline D.Watson & 1.955537 & 2.013548 & 1.648153 & 2.025907 \\
\hline
\end{tabular}

Short run estimates show that demand has a significant effect on the prices of Ethereum and Ripple. Oil prices also show effect on the prices of Ripple. Rest of the hypotheses are not supported in any of the coin analysis.

\subsection{Long run analysis}

\begin{tabular}{|l|l|l|}
\hline VARIABLES & Ethereum & Ripple \\
\hline
\end{tabular}




\begin{tabular}{|l|l|l|}
\hline Supply & $1.03 \mathrm{E}-08^{* * *}$ & $2.56 \mathrm{E}-11^{* * *}$ \\
\hline Demand & $3.87 \mathrm{E}-11$ & $2.12 \mathrm{E}-12^{* * *}$ \\
\hline Attractiveness & $2.625471^{* * *}$ & 0.001284 \\
\hline Stock Prices & -0.007359 & $7.46 \mathrm{E}-05$ \\
\hline Exchange Rate & $-20.86419^{* * *}$ & -0.004329 \\
\hline Oil Prices & -0.073589 & -0.001687 \\
\hline Constant & -3.411942 & 0.075565 \\
\hline F-Statistics & $538074.8^{* * *}$ & $3412072^{* * *}$ \\
\hline D.Watson test & 1.924238 & 2.10678 \\
\hline
\end{tabular}

The table above shows that our first hypothesis is supported in Ethereum and Ripple crypto coins. The first hypothesis was the significant effect of supply on crypto prices. Second hypothesis of significant effect of demand on the prices of digital currencies has been supported in Ripple only. This means that only the Ripple prices are driven by the market force of demand and supply. Third hypothesis of the effect of attractiveness is supported in Ethereum analysis. This indicates that Ethereum is a source of attraction to investors. Fifth hypothesis of exchange rate effect on crypto prices is supported in Ethereum analysis. Sixth hypothesis of oil prices effect is not present in any of the coin price estimation.

\section{CONCLUSION AND RECOMMENDATIONS}

\subsection{Conclusion}

From our analysis we may be able to conclude that Ethereum and Ripple coin's price movement is mainly and significantly driven by their circulating supply. Pavel Ciaian (2016) proved that Bitcoin price is driven mainly by supply and demand. Whereas in our study Ethereum and Ripple support that. Ethereum is gaining attention after Bitcoin. Exchange rates also effect Ethereum prices in Pakistan. Whereas, Bitcoin analysis became insignificant in all the cases of long run. This is because Bitcoin has a fixed supply of coins to be mined and it has almost reached its full capacity. Our analysis 
also concludes that cryptocoins do not go through the main financial system of Pakistan except for Ripple which has been used by banks and financial institutions to facilitate their transactions.

On one hand, the whole worldwide money related framework is ending up progressively digitized; on another, printing paper and coins is a costly procedure that may one day wind up out of date. Additionally, individuals are utilizing the web increasingly for retail acquiring and somebody needs to give the methods for exchanging the cash. Generally, digital forms of money have far to go before they can supplant charge cards and conventional monetary standards as an instrument for worldwide trade. Numerous individuals are as yet ignorant of digital currency and should be instructed about it to have the capacity to apply it to their lives. Organizations need to begin tolerating it. They have to make it simpler to join and begin.

\subsection{Recommendations}

For future references it is recommended that comparative analysis of different countries should be done in order to better understand the price drivers of cryptocurrencies in different regions of the world. This would help the policy makers to fully utilize this new tech virtual phenomenon.

\subsection{Limitations}

The main limitation of this study is the unavailability of sufficient data for this new phenomenon. Being a recent technology, not much research has been done on cryptocurrencies. Also the data available starts from the year 2013 for Bitcoin and the other Altcoins from 2015 onwards.

\section{References}


Abeer ElBahrawya, L. A.-S. (2017). Evolutionary dynamics of the cryptocurrency market. physics.soc-ph.

Baek, C., \& Elbeck, M. (2015). Bitcoins as an investment or speculative vehicle? A first look, Applied Economics Letters, 22:1, 30-34. doi:10.1080/13504851.2014.916379

Baur, D. G., \& Dimplf, T. (2021). The volatility of Bitcoin and its role as a medium of exchange and a store of value. Empirical economics, Springer.

Brue, M. (2001). Microeconomics (16th ed.). McGraw Hill.

Btcpk.net. (2020, May 14). Retrieved April 14, 2018, from Btcpk.net: https://www.btcpk.net/

Coingecko. (2020, 07 01). Retrieved from Coingecko.com: https://www.coingecko.com/en

Coinmarket. (2020, 07 07). Retrieved from Coinmarket.com: https://coinmarketcap.com/

Dyhrberg, A. H. (2015). Hedging Capabilities of Bitcoin. Is it the virtual gold? University College Dublin. School of Economics, 14.

Folkinshteyn, D. \&. (2015). The Bitcoin Mirage. Journal of Strategic and International Studies. X.

Frederik Armknecht, G. O. (2016). Ripple: Overview and Outlook. Germany.

Galina Hale, A. K. (2018, December). FRBSF Economic Letter. Retrieved January 10, 2019, from frbsf: https://www.frbsf.org/economic-research/files/el2018-12.pdf

Graeber, D. (2011). Debt The First 5,000 Years. Melville House Publishing, 542.

Hagman, M. (2014). Money Management Using the Kelly Criterion. UMEA University, 34.

Kaminski, J. C. (2014). Nowcasting the Bitcoin Market with Twitter signals. MIT Media Lab.

Karafiloski, E. (2017). Blockchain Solutions for Big Data Challenges. Faculty of Computer Science and Engineering,Ss. Cyril and Methodius University,Skopje, Macedonia, 1-6.

Katsiampa, P. (2017). Volatility estimation for Bitcoin. Elsevier.

Kristoufek, L. (2013). What Are the Main Drivers of the Bitcoin. doi:10.1371/journal.pone.0123923

Lee, A. D. (2014). Bitcoin: Currency or Investment? ssrn.

Mankiw, N. G. (2006). Principles of microeconomics (6th ed.). south western publishers.

Martis Buchholz, J. D. (2012). Bits and Bets Information, Price Volatility, and Demand for Bitcoin. Economics 312, Retrieved 10/10/2018, https://www.reed.edu/economics/parker/s12/312/finalproj/Bitcoin.pdf

Mehta, A. A. (2017). Impact of Bitcoin as a World Currency. Accounting and Finance Research, 117.

Morisse, M. (2015). Cryptocurrencies and Bitcoin: Charting the Research Landscape. Department of Informatics University of Hamburg, 1-16. 
Nakamoto, s. (2009). Bitcoin: A Peer-to-Peer Electronic Cash System. 9. www.bitcoin.org.

Odean, B. M. (2006). All that Glitters: The Effect of Attention and News on the Buying Behavior of Individual and Institutional Investors. The Review of Financial Studies.

Pavel Ciaian, M. R. (2016, February 2). The digital agenda of virtual currencies: Can BitCoin become a global currency? 37. springerlink.com. doi: 10.1007/s10257-016-0304-0

Peng, M. W. (2009). How do internal capabilities and external partnerships affect innovativeness? https://link.springer.com/journal/10490.

Pesaran, M. H. (1999). bounds testing approaches to the analysis of long run relationships.

Pesaran, M. H. (1999). Bounds testing approaches to the analysis of long run relationships.

Poyser, O. (2017). Exploring the determinants of Bitcoin's price.

Rainer Böhme, N. C. (2015). Bitcoin: Economics, Technology, and Governance. Journal of Economic Perspectives_Volume 29, Number 2-Spring 2015-Pages 213-238, 1-32.

Rauchs, D. G. (2017). Global cryptocurrency benchmarking study. University of Cambridge, Judge business school, UK.

Rituparna Ghosh, K. H. (2016). Bitcoin or Ethereum? The million dollar question. Carey business school, Johns Hopkins.

Růstu, 1. R. (2016, 3 16). Straight line growth rate. Retrieved 5 17, 2018, from Febmat: https://www.febmat.com/en/article-straight-line-growth-rate/

Salvatore, D. (n.d.). Managerial economics in a global economy (7th ed.). Fordham University.

Sauer, B. (2016, April 26). Virtual Currencies, the Money Market, and Monetary policy. International Atlantic Economic Society 2016. doi:10.1007/s11294-016-9576-X

Selmi, J. B. (2015). What Does Bitcoin Look Like? Annals of Economics and Finance, 45. Retrieved 10/10/2018, from https://www.researchgate.net/publication/283676718

Sovbetov, Y. (2018). Factors Influencing Cryptocurrency Prices: Evidence from Bitcoin, Ethereum, Dash, Litcoin, and Monero. Journal of Economics and Financial Analysis, Vol:2, No:2 (2018) 1-27, 1-28.

Teigland, R. \&. (2013). Breaking Out of the Bank in Europe - Exploring Collective Emergent Institutional Entrepreneurship Through Bitcoin. SSRN Electronic Journal(10.2139/ssrn.2263707).

Vergne, S.W.P. (2017). Buzz Factor or Innovation Potential:. plos one. doi:10.1371/journal.pone.0169556

Veronica Greco, D. R. (2001). Coping with uncertainty. Personality and individual differences. doi:31(4):519-534

Vetro`, M. C. (2018). Blockchain for the Internet of Things: a Systematic Literature Review. 
Politecnico di torino Repositoryistituzionale, 1-7.

Wijk, D. v. (2013). What can be expected from the Bitcoin? Erasmus Universiteit Rotterdam(345986), 21.

Wilson, A. Y. (2015). Characteristics of Bitcoin users. doi:10.1080/13504851.2014.995359

Yermack, D. (2015). Is bitcoin a real currency. National Bureau of Economic Research (19747). 